\title{
ISOMORPHIC EXTENSIONS AND APPLICATIONS
}

\author{
TOMASZ DOWNAROWICZ AND ELI GLASNER
}

\begin{abstract}
If $\pi:(X, T) \rightarrow(Z, S)$ is a topological factor map between uniquely ergodic topological dynamical systems, then $(X, T)$ is called an isomorphic extension of $(Z, S)$ if $\pi$ is also a measure-theoretic isomorphism. We consider the case when the systems are minimal and we pay special attention to equicontinuous $(Z, S)$. We first establish a characterization of this type of isomorphic extensions in terms of mean equicontinuity, and then show that an isomorphic extension need not be almost one-to-one, answering questions of $\mathrm{Li}, \mathrm{Tu}$ and $\mathrm{Ye}$.
\end{abstract}

\section{INTRODUCTION}

Throughout, by a topological dynamical system (denoted $(X, T)$, or similarly) we will always mean the action of a homeomorphism $T$ on an infinite compact metric space $X$. Although many of our results apply to the noninvertible case, for simplicity, we focus on invertible systems only.

By a topological model of an (invertible) ergodic measure-preserving transformation (m.p.t. for short) $(\Omega, \mathcal{F}, \nu, S)$, we will mean any uniquely ergodic topological system $(X, T)$ (with the unique invariant measure $\mu$ ), such that $(X$, $\operatorname{Borel}(X), \mu, T)$ and $(\Omega, \mathcal{F}, \nu, S)$ are measure-theoretically isomorphic.

The celebrated Jewett-Krieger theorem asserts that every invertible ergodic m.p.t. has a strictly ergodic (i.e. minimal and uniquely ergodic) topological model. In this work, we are interested in the relations between various strictly ergodic models of the same ergodic system. More specifically we will focus on the situation when one model is a topological extension of another. Also, of particular interest to us is the case when the underlying m.p.t. belongs to the class of the simplest ergodic systems, namely those with discrete spectrum. Recall that a "standard model" of a discrete spectrum ergodic system has the form of a rotation, by a topological generator, on a compact monothetic group or, equivalently, is a minimal equicontinuous system (Halmos-von Neumann Theorem, see e.g. [9, Chapter 1, Section 2]).

We will provide a natural classification of all the strictly ergodic topological models of an ergodic system with discrete spectrum which topologically extend the standard model. Before we formulate our results, we need to establish some basic terminology. We will assume that the reader is familiar with the textbook notions of a factor (in particular, maximal equicontinuous factor), extension, isomorphism, ergodicity,

Date: February 6, 2015.

2010 Mathematics Subject Classification. 37xx, 37A05, 37B05.

Key words and phrases. minimality, unique ergodicity, isomorphic extension, almost one-to-one extension, mean equicontinuity, skew product.

The research of the first named author is supported by the NCN (National Science Center, Poland) grant 2013/08/A/ST1/00275. The research of the second named author was supported by a grant of ISF $668 / 13$. 
minimality, discrete spectrum, etc., as well as with the definition of lower and upper Banach density of a subset of integers and some related notions. When dealing with an m.p.t. which arises from a topological dynamical system equipped with an invariant probability measure, we will always assume that the sigma-algebra in question is the Borel sigma-algebra completed with respect to that measure (and skip it in the notation of the system). The reader is referred to Furstenberg's classical monograph [5], or to [9] for more details and background.

Most of the time we will consider a pair of uniquely ergodic topological dynamical systems $(X, T)$ and $(Z, S)$. In such a case, $\mu$ and $\nu$ will always denote the unique invariant measures on $X$ and $Z$, respectively. Notice that a topological factor of a uniquely ergodic system is uniquely ergodic as well.

Definition 1.1. We say that $(X, T)$ is an isomorphic extension of $(Z, S)$ if $(X, T)$ is uniquely ergodic and there exists a topological factor map $\pi: X \rightarrow Z$ which is, at the same time, a measure-theoretic isomorphism between $(X, \mu, T)$ and $(Z, \nu, S)$.

It is clear that an isomorphic extension of a topological model of some (ergodic) m.p.t. is another topological model of the same m.p.t. Notice that the requirement in the definition is stronger than just assuming that $(X, T)$ is a topological extension of $(Z, S)$ and that the systems $(X, \mu, T)$ and $(Z, \nu, S)$ are measure-theoretically isomorphic. The measure-theoretic isomorphism must be realized by the same map which establishes the topological factor relation. Isomorphic extensions are important because they carry over many "hybrid properties" of the base system $(Z, S)$ to the extended system $(X, T)$. The adjective "hybrid" refers to properties which combine measure-theoretic and topological notions. As an example recall the notion of uncorrelation: two bounded complex-valued sequences $\left(x_{n}\right)_{n \geq 1}$ and $\left(y_{n}\right)_{n \geq 1}$ are called uncorrelated if

$$
\lim _{n}\left|\frac{1}{n} \sum_{i=1}^{n} x_{i} \bar{y}_{i}-\left(\frac{1}{n} \sum_{i=1}^{n} x_{i}\right)\left(\frac{1}{n} \sum_{i=1}^{n} \bar{y}_{i}\right)\right|=0 .
$$

Sarnak's conjecture asserts that any topological dynamical system $(X, T)$ with zero topological entropy satisfies the following uncorrelation condition:

Fix an arbitrary continuous (complex-valued) function $f$ on $X$ and any point $x \in X$. Then, the sequence $\left(a_{n}\right)_{n \geq 1}$ defined by: $a_{n}=f\left(T^{n} x\right)$ is uncorrelated to the Möbius function $\left(\mu_{n}\right)_{n \geq 1}$ (see [22] for more details).

In [3, Theorem 4.1] it is proved that if a topological system $(Z, S)$ fulfills the uncorrelation condition and $(X, T)$ is an isomorphic extension of $(Z, S)$ then $(X, T)$ also fulfills the uncorrelation condition. In fact, the proof does not rely on any specific property of the Möbius function; it shows that isomorphic extensions preserve the property of uncorrelation with respect to any fixed bounded complex-valued sequence.

Since minimal rotations of compact monothetic groups fulfill the uncorrelation condition, it follows from [3] that every isomorphic extension of the standard model of an ergodic system with discrete spectrum also fulfills the uncorrelation condition ${ }^{1}$.

\footnotetext{
${ }^{1}$ Let us mention that recently, H. El Abdalaoui, M. Lemańczyk and T. de la Rue (private communication) proved that any topological model of an ergodic system with irrational discrete spectrum satisfies Sarnak's uncorrelation condition. It is striking that even for the system with the rational discrete spectrum $\{-1,1\}$ (whose standard model is the two-point periodic system) the validity of the conjecture for all (uniquely ergodic) topological models remains undecided.
} 
An isomorphic extension can be described as one given by a topological factor map $\pi: X \rightarrow Z$ which becomes invertible after discarding from both spaces subsets of measure zero. By analogy, one can require that the map $\pi$ becomes invertible after discarding from both spaces some meager (first category) sets. This leads to the notion of an almost 1-1 extension, which can be regarded as a topological analog of an isomorphic extension (how good is this analogy - will become clear from what follows):

Definition 1.2. We say that $(X, T)$ is an almost one-to-one (almost 1-1) extension of $(Z, S)$ if there exists a topological factor map $\pi: X \rightarrow Z$ such that the union of the singleton fibers is dense in $X$. If $(X, T)$ is minimal it suffices that at least one singleton fiber exists.

By invariance, for any ergodic measure on $X$, the union of singleton fibers has measure either zero or one. Thus, uniquely ergodic almost 1-1 extensions can be classified as follows (note that these are "hybrid" notions):

Definition 1.3. Let $(X, T)$ be a uniquely ergodic system which is an almost 1-1 extension of $(Z, S)$. We say that this extension is regular if the union of the singleton fibers has full $\mu$ measure. Otherwise, when this union has measure zero, the extension is called irregular.

It is obvious that a regular almost 1-1 extension is automatically isomorphic. On the other hand, it is well-known that irregular almost 1-1 extensions are usually far from being isomorphic and many invariants need not be preserved. For instance the extension may have much richer spectral properties, larger entropy, many more invariant measures, etc. So, two natural questions arise: can an irregular almost 1-1 extension still be isomorphic? And, can an isomorphic extension be not almost 1-1 at all? The first question has a positive answer; an appropriate example is provided in [3, Example 5.1]. Another example of this phenomenon is to be found in the work of Kerr and Li [17, Section 11], see Remark 5.2. in [10]. In the present paper we will provide a positive answer to the second question, as well, already in the class of minimal systems. As a result, we obtain the following classification of isomorphic extensions (ordered decreasingly with respect to "topological closeness"), where all three, mutually disjoint classes, are nonempty:

1. regular almost 1-1 extensions,

2. irregular, yet isomorphic, almost 1-1 extensions,

3. isomorphic, but not even almost 1-1 extensions.

For example, if $(Z, S)$ is a standard model of an ergodic m.p.t. with infinite discrete spectrum, we obtain three types of strictly ergodic topological models with subtly different topological properties.

Let us mention that there exist strictly ergodic topological models of ergodic systems with infinite discrete spectrum which are topologically much removed from the standard models. For instance, there exist such models which are topologically weakly (or even srtongly) mixing, in which case there is no topological factor map in either direction, see e.g. [18] or [13].

It turns out that isomorphic extensions can also be used to characterize the classes of systems called mean equicontinuous and Weyl mean equicontinuous. 
Definition 1.4. If $(X, T)$ is a dynamical system, we define the Besicovitch and Weyl distances between points, respectively, as

$$
\begin{aligned}
& d_{\mathrm{B}}(x, y):=\limsup _{n \rightarrow \infty} \frac{1}{n} \sum_{i=0}^{n-1} d\left(T^{i} x, T^{i} y\right), \\
& d_{\mathrm{W}}(x, y):=\limsup _{n-m \rightarrow \infty} \frac{1}{n-m} \sum_{i=m}^{n-1} d\left(T^{i} x, T^{i} y\right) .
\end{aligned}
$$

A system $(X, T)$ is called mean equicontinuous or Weyl mean equicontinuous ${ }^{2}$ if for every $\epsilon>0$ there exists $\delta>0$ such that

$$
d(x, y)<\delta \Longrightarrow d_{\mathrm{B}}(x, y)<\epsilon, \quad d(x, y)<\delta \Longrightarrow d_{\mathrm{W}}(x, y)<\epsilon,
$$

respectively.

It is easy to see that the Besicovitch and Weyl distances are invariant pseudometrics. Mean equicontinuity is continuity of the quotient map $(X, d) \rightarrow\left(X / \approx, d_{\mathrm{B}}\right)$, where $\approx$ is the mean asymptotic relation $x \approx y \Longleftrightarrow d_{\mathrm{B}}(x, y)=0$. An analogous statement with $d_{\mathrm{B}}$ replaced by $d_{\mathrm{W}}$ applies to Weyl mean equicontinuity.

$\mathrm{Li}$, $\mathrm{Tu}$ and Ye [19] posed the following questions, based on observation of the examples which were available to them:

Question 1.5. Is every minimal mean equicontinuous system also Weyl mean equicontinuous?

Question 1.6. Is every minimal Weyl mean equicontinuous system an almost 1-1 extension of its maximal equicontinuous factor?

In the next two sections we prove theorems which allow us to answer Question 1.5 positively (Theorem 2.1), then we reduce Question 1.6 to a problem concerning isomorphic extensions, and finally, answer this question negatively (Theorem 3.1). In the last section we present some concrete instances of our main result and refer to some related topics in the literature.

\section{A CHARACTERIZATION OF MINIMAL MEAN EQUiCONTINUOUS SYSTEMS}

Theorem 2.1. Let $(X, T)$ be a minimal topological dynamical system with maximal equicontinuous factor $(Z, S)$. Then the following are equivalent

1. $(X, T)$ is mean equicontinuous,

2. $(X, T)$ is Weyl mean equicontinuous,

3. $(X, T)$ is an isomorphic extension of $(Z, S)$ (in particular, $(X, T)$ is then uniquely ergodic).

Remarks 2.2. $\quad$ a. Note that if $(X, T)$ is an isomorphic extension of a minimal equicontinuous system $(Z, S)$, then $(Z, S)$ is necessarily its maximal equicontinuous factor.

\footnotetext{
${ }^{2}$ In [19] the authors call this notion Banach mean equicontinuous (probably referring to the notion of upper Banach density). We believe that the reference to Weyl is more appropriate here.
} 
b. Huang, Lu and Ye [16] introduce a hybrid notion of $\mu$-equicontinuity (of a topological system with a fixed invariant measure $\mu$ ) and prove that this property implies discrete spectrum of $(X, \mu, T)$. Garcia-Ramos [8] proves that an even weaker (hybrid) property, $\mu$-mean equicontinuity, is in fact equivalent to discrete spectrum of $(X, \mu, T)$. The implication $1 . \Longrightarrow 3$. above (which is also implicit in the proof of Theorem 3.8 of [19]) has the same flavor; it implies that mean equicontinuous systems are uniquely ergodic with discrete spectrum. Clearly, the condition 3. gives more specific information about the system.

Proof of the theorem. We let $\pi: X \rightarrow Z$ denote the maximal equicontinuous factor map.

1. $\Rightarrow 3 .:$ Let $(X, T)$ be mean equicontinuous. First we show that two points which are in the same fiber over the maximal equicontinuous factor are mean asymptotic (i.e., the Besicovitch distance between them is zero).

By a theorem of Veech [25] (see also [2]) such points, say $x, y$, are regionally proximal, i.e., there are sequences $n_{k}, x_{k}, y_{k}$ with $x_{k} \rightarrow x, y_{k} \rightarrow y$ and $d\left(T^{n_{k}} x_{k}, T^{n_{k}} y_{k}\right) \rightarrow$ 0 . Given $\epsilon>0$ let $\delta$ be as in the definition of mean equicontinuity. For a sufficiently large $k$ we have:

$$
\begin{aligned}
& d\left(x_{k}, x\right)<\delta, \text { hence } d_{\mathrm{B}}\left(x_{k}, x\right)<\epsilon, \\
& d\left(y_{k}, y\right)<\delta, \text { hence } d_{\mathrm{B}}\left(y_{k}, y\right)<\epsilon, \\
& d\left(T^{n_{k}} x_{k}, T^{n_{k}} y_{k}\right)<\delta, \text { hence } d_{\mathrm{B}}\left(x_{k}, y_{k}\right)=d_{\mathrm{B}}\left(T^{n_{k}} x_{k}, T^{n_{k}} y_{k}\right)<\epsilon .
\end{aligned}
$$

We have shown that $d_{\mathrm{B}}(x, y)<3 \epsilon$ for every $\epsilon>0$, hence $d_{\mathrm{B}}(x, y)=0$.

As $(Z, S)$ is minimal and equicontinuous, it is strictly ergodic. Suppose now that $\pi$ is not an isomorphic extension. This means that on $X$ there are either (at least) two distinct ergodic measures $\mu, \mu^{\prime}$, or there is just one ergodic measure $\mu$, but its disintegration over $\nu$ produces fiber measures which, with positive $\nu$ probability, are not point masses.

In the first case we take any joining $\xi$ of $\mu$ with $\mu^{\prime}$ over the common factor $\nu$, in the other case we take the relatively independent self-joining of $\mu$ with itself over the common factor $\nu$ (we set $\mu^{\prime}=\mu$ ). In either case $\xi$ is not concentrated on the diagonal. Becuase both $\mu$ and $\mu^{\prime}$ are ergodic, $\xi$ decomposes into ergodic joinings. In either case we can find an ergodic component $\xi^{\prime}$ of $\xi$ which not concentrated on the diagonal. The push-forward measure $(\pi \times \pi)_{*}(\xi)$ and hence also $(\pi \times \pi)_{*}\left(\xi^{\prime}\right)$ is the identity selfjoining of $\nu$ (this is the meaning of the fact that our joinings were "over the common factor $\left.\nu^{\prime \prime}\right)$. Since $\xi^{\prime}$-almost every pair $(x, y)$ is generic for $\xi^{\prime}$, and $\xi^{\prime}$-almost every pair $(x, y)$ satisfies $\pi x=\pi y$, we can find a pair $(x, y)$ generic for $\xi^{\prime}$, such that $x$ and $y$ are in the same fiber. On the other hand, since $\xi^{\prime}$ is not supported by the diagonal, there is a closed $\epsilon$-neighborhood of the diagonal whose open complement has positive $\xi^{\prime}$ measure, say $\gamma>0$. As $(x, y)$ is generic, the orbit of $(x, y)$ visits this complement with lower density larger than $\gamma$. Thus, $d_{\mathrm{B}}(x, y)>\epsilon \gamma>0$, and this contradicts the fact that the pair $x, y$ is mean asymptotic.

3. $\Rightarrow 2 .:$ Now suppose that $(X, T)$ is a (uniquely ergodic) isomorphic extension of $(Z, S)$. Let $\pi$ be the factor map from $X$ onto $Z$ (which is also a measure-theoretic isomorphism). On $Z$ we choose a metric $d$ for which $S$ is an isometry. Moreover, we can assume that the metric on $X$ (also denoted by $d$ ) satisfies $d(x, y) \geq d(\pi x, \pi y)$. 
The map $\pi$ becomes invertible after discarding a null set in $X$ and a null set in $Z$. The inverse function $\pi^{-1}: Z \rightarrow X$ (defined almost everywhere on $Z$ ) is measurable, hence, by Luzin's theorem, continuous when restricted to some subset $A \subset Z$ of large $\nu$ measure, say $\nu(A)>1-\epsilon$. By the regularity of $\nu$ we can assume that $A$ is closed, hence compact. Let $f: A \rightarrow X$ denote the restriction $\left.\pi^{-1}\right|_{A}$. Let $U$ be the $\delta$-neighborhood of $f(A)$, where $\delta$ will be specified later. Clearly, $\mu(U) \geq \mu(f(A))=\nu(A)>1-\epsilon$.

Take two points $x, y \in X$ with $d(x, y)<\delta$ and observe their forward orbits. First note that $d(\pi x, \pi y)<\delta$ and, because the map $S$ is an isometry, the corresponding points on their orbits remain at the same distance. By the unique ergodicity of $(X, T)$, the points $x$ and $y$ are uniformly generic ${ }^{3}$ for $\mu$. In particular, their forward orbits visit $U$ with lower Banach densities larger than $1-\epsilon$. Hence, with lower Banach density at least $1-2 \epsilon$ they fall simultaneously in $U$.

Consider an $n$ such that both $T^{n} x, T^{n} y$ fall in $U$. Then, there are points $x^{\prime}, y^{\prime} \in$ $f(A)$ such that $d\left(T^{n} x, x^{\prime}\right)<\delta, d\left(T^{n} y, y^{\prime}\right)<\delta$. This implies that

$$
\begin{array}{r}
d\left(\pi x^{\prime}, \pi y^{\prime}\right) \leq d\left(\pi x^{\prime}, \pi T^{n} x\right)+d\left(\pi T^{n} x, \pi T^{n} y\right)+d\left(\pi T^{n} y, \pi y^{\prime}\right) \leq \\
d\left(x^{\prime}, T^{n} x\right)+d\left(S^{n} \pi x, S^{n} \pi y\right)+d\left(T^{n} y, y^{\prime}\right) \leq 3 \delta .
\end{array}
$$

Since $x^{\prime}=f\left(\pi x^{\prime}\right), y^{\prime}=f\left(\pi y^{\prime}\right)$, and $f$ is uniformly continuous on $A$, for a suitably small $\delta<\frac{\epsilon}{4}$ we have $d\left(x^{\prime}, y^{\prime}\right)<\frac{\epsilon}{2}$, and thus

$$
d\left(T^{n} x, T^{n} y\right) \leq d\left(T^{n} x, x^{\prime}\right)+d\left(x^{\prime}, y^{\prime}\right)+d\left(y^{\prime}, T^{n} y\right)<\delta+\frac{\epsilon}{2}+\delta<\epsilon .
$$

We have shown that given $\epsilon>0$ there exists $\delta>0$ such that $d(x, y)<\delta$ implies $d\left(T^{n} x, T^{n} y\right)<\epsilon$ for $n$ 's in a set of lower Banach density at least $1-\epsilon$. But that is exactly the meaning of Weyl mean equicontinuity of $(X, T)$.

The implication $2 . \Rightarrow 1$. is trivial.

The above theorem not only answers Question 1.5, but also allows us to formulate Question 1.6 in an equivalent way which does not refer to the notion of mean equicontinuity:

Question 2.3. Let $(X, T)$ be a minimal system and let $(Z, S)$ be its maximal equicontinuous factor via a map $\pi: X \rightarrow Z$. Suppose that $(X, T)$ is an isomorphic extension of $(Z, S)$, is $\pi$ necessarily almost one-to-one?

As already mentioned, we will answer this question negatively. In fact, in the next section we will prove a much more general result, where isomorphic non almost one-to-one extensions will be shown to be generic ${ }^{4}$ in a certain setup.

We take this opportunity to investigate the minimal size of a fiber in a topological extension. A priori, the general semicontinuity properties of the "fiber size function"

\footnotetext{
${ }^{3}$ In a uniquely ergodic system $(X, T)$ every point $x$ is uniformly generic, which means that the limit$$
\lim _{n-m \rightarrow \infty} \frac{1}{n-m} \sum_{i=m}^{n-1} f\left(T^{i} x\right)
$$

exists for every continuous function $f$ and equals the integral of $f$.

${ }^{4}$ Here and in the sequel, the term "generic" refers to "belonging to a residual subset", where a residual subset of a Polish space $X$ is a set which contains a dense $G_{\delta}$ subset of $X$. Not to be confused with "generic points" for an invariant measure.
} 
do not suffice to draw the conclusion that in the non-almost 1-1 case the infimum of this function is positive ${ }^{5}$, hence the lemma below may be of interest.

Lemma 2.4. Let $\pi:(X, T) \rightarrow(Z, S)$ be a topological factor map, where $X$ (and hence also $Z)$ is minimal. Define diam $: Z \rightarrow[0, \operatorname{diam}(X)]$ by $\operatorname{diam}(z)=\operatorname{diam}\left(\pi^{-1}(z)\right)$. Then either $\pi$ is almost 1-1 (in which case $\inf (\operatorname{diam})=\min (\operatorname{diam})=0$ ) or $\inf (\operatorname{diam})>0$.

Proof. Suppose $\pi$ is not almost 1-1. Then diam is positive everywhere on $Z$. Pick a decreasing to zero sequence $\left(\delta_{n}\right)$, such that $d\left(x, x^{\prime}\right)<\delta_{n+1} \Longrightarrow d\left(T x, T x^{\prime}\right)<\delta_{n}$ (here $d$ represents the metric on $X)$. Because $S$ is a homeomorphism, we have $z=S^{-1} S z$ and hence

$$
\pi^{-1}(z)=\pi^{-1} S^{-1} S z=T^{-1} \pi^{-1} S z
$$

which implies that each set $A_{n+1}=\left\{y: \operatorname{diam}(y)<\delta_{n+1}\right\}$ is mapped by $S$ into $A_{n}$. Notice that all the sets $A_{n}$ are open. If all these sets were nonempty, then each of them would contain arbitrarily long pieces of orbits, which, by minimality, would imply that all these sets were dense. By the Baire category theorem their intersection would be nonempty, implying that $\operatorname{diam}(z)=0$ at some point, i.e., the extension would be almost 1-1. We have shown that indeed diam is bounded from below by some positive constant.

We now prove another characterization of isomorphic extensions which will become useful in the following section. Given a factor map between dynamical systems $\pi$ : $(X, T) \rightarrow(Z, S)$, we form an associated system, $W=X \underset{Z}{\times} X=\left\{\left(x, x^{\prime}\right) \in X \times X:\right.$ $\left.\pi(x)=\pi\left(x^{\prime}\right)\right\}$, with the diagonal action $(T \times T)\left(x, x^{\prime}\right)=\left(T x, T x^{\prime}\right)$. We call $W$ the relative product of $X$ over $Z$. We then have:

Proposition 2.5. A system $(X, T)$ is an isomorphic extension of $(Z, S)$ if and only if there exists a topological factor map $\pi:(X, T) \rightarrow(Z, S)$ such that the associated relative product $(W, T \times T)$ is uniquely ergodic.

Proof. $(\Leftarrow)$ : First observe that, since $(X, T)$ is a factor of $(W, T)$, it is uniquely ergodic. As usual, we will denote the invariant measure on $X$ by $\mu$ and the one on $Z$ by $\nu$. Next observe that on $W$ there are always two obvious $(T \times T)$-invariant measures, namely the diagonal measure $\mu_{\Delta}=J_{*}(\mu)$, where $J: X \rightarrow W$ is the map $J(x)=(x, x)$, and the relative product measure $\mu \underset{\nu}{\times} \mu$, which is obtained as follows: Let $\mu=\int \mu_{z} d \nu(z)$ be the disintegration of $\mu$ over $\nu$, then

$$
\mu \underset{\nu}{\times} \mu:=\int\left(\mu_{z} \times \mu_{z}\right) d \nu(z) .
$$

Now the fact that $(W, T)$ is uniquely ergodic implies that $\mu_{\Delta}=\mu \times \mu$, i.e. disintegrating the left hand side over $\nu$, we get

$$
\int\left(\mu_{\Delta}\right)_{z} d \nu(z)=\int\left(\mu_{z} \times \mu_{z}\right) d \nu(z)
$$

By uniqueness of the disintegration, we have $\left(\mu_{\Delta}\right)_{z}=\mu_{z} \times \mu_{z}$ for $\nu$-a.e. $z$.

\footnotetext{
${ }^{5}$ The function diam defined in the proof is upper (and not lower) semicontinuous, thus it need not achieve its minimum.
} 
Clearly, $\nu$-a.e. $\left(\mu_{\Delta}\right)_{z}$ is supported on the diagonal set $\Delta_{z}=\left\{(x, x): x \in \pi^{-1}(z)\right\}$, while the only measures on $\pi^{-1}(z)$ whose Cartesian square is supported by this diagonal set are point masses. We deduce that $\nu$-a.e. $\mu_{z}$ is a point mass. Denoting by $\varphi(z) \in \pi^{-1}(z)$ the atom of $\mu_{z}$, we easily see that $\varphi: Z \rightarrow X$ is a measure-theoretic isomorphism whose inverse (wherever defined) coincides with $\pi$.

$(\Rightarrow)$ : We now assume that $(X, T)$ is an isomorphic extension of $(Z, S)$, i.e., that there exists a $\nu$-a.e. defined measurable bijection $\varphi: Z \rightarrow X$, whose inverse coincides with $\pi$ (i.e., $\varphi(z) \in \pi^{-1}(z)$, $\nu$-a.s.), and such that $\mu=\varphi_{*}(\nu)$ is a unique invariant measure on $X$. Clearly, the disintegration of $\mu$ over $\nu$ is into point masses on the graph of $\varphi$ :

$$
\mu=\int \delta_{\varphi(z)} d \nu(z)
$$

Let now $\lambda$ be a $T \times T$-invariant measure on $W$. It disintegrates over $\nu$ as

$$
\lambda=\int \lambda_{z} d \nu(z)
$$

where $\nu$-a.e. $\lambda_{z}$ is supported by the fiber of $z$ in $W$, i.e., by $\pi^{-1}(z) \times \pi^{-1}(z)$. Letting $\lambda_{i, z}(i=1,2)$ denote the marginals of $\lambda_{z}$ on the two copies of $\pi^{-1}(z)$, respectively, we can see that both measures $\int \lambda_{i, z} d \nu(z)$ are invariant measures on $X$. So, both of them must equal $\mu$ and, by uniqueness of the disintegration, for $\nu$-a.e. $z$ we have

$$
\lambda_{1, z}=\lambda_{2, z}=\delta_{\varphi(z)}
$$

hence $\lambda_{z}=\delta_{\varphi(z)} \times \delta_{\varphi(z)}=\delta_{(\varphi(z), \varphi(z))}$. This proves uniqueness of $\lambda$.

\section{A generic extension in $\overline{\operatorname{Cob}}(\mathcal{G})$ Is isomorphic}

Our purpose in this section is to show the existence of isomorphic extensions which are not almost one-to-one. Given a strictly ergodic topological system $(Z, S)$, we will investigate a certain associated class of skew product extensions, with an appropriate fiber space $Y$.

Let $Y$ be a (sufficiently rich) compact metric space, and let $\mathcal{G}$ be a closed subgroup of the group of all homeomorphisms of $Y$, equipped with the uniform metric (this topology makes $\mathcal{G}$ a Polish topological group). Let $C(Z, \mathcal{G})$ be the family of all continuous maps $G: Z \rightarrow \mathcal{G}$, which we will call cocycles. We will write $G_{z}$ (rather than $G(z))$ to denote the homeomorphism of $Y$ associated to $z$, while $G_{z}(y)$ will denote its value at $y \in Y$. Equipped with the uniform topology, $C(Z, \mathcal{G})$ is again a Polish group, with multiplication and inverse defined poinwise: $(G H)_{z}=G_{z} H_{z},\left(G^{-1}\right)_{z}=\left(G_{z}\right)^{-1}$.

From a cocycle $G \in C(Z, \mathcal{G})$ we can create a coboundary $G_{S}^{-1} G$, where $G_{S}$ is defined by $\left(G_{S}\right)_{z}=G_{S z}$ (note that $\left(G_{S}\right)^{-1}=\left(G^{-1}\right)_{S}$ hence one can skip the parentheses). Thus we have $\left(G_{S}^{-1} G\right)_{z}(y)=G_{S z}^{-1}\left(G_{z}(y)\right)$. Clearly, the coboundary still belongs to $C(Z, \mathcal{G})$. The collection of all coboundaries obtained in this manner will be denoted by $\operatorname{Cob}(\mathcal{G})$. In general, this is neither a subgroup nor a closed subset of $C(Z, \mathcal{G})$, so

we will work with the closure $\overline{\operatorname{Cob}}(\mathcal{G})$, which is just a Polish space (enough to use category arguments). 
With each cocycle $G \in C(Z, \mathcal{G})$ we associate a skew product extension of $(Z, S)$ defined on the product space $X=Z \times Y$ by:

$$
T_{G}(z, y)=\left(S z, G_{z}(y)\right)
$$

We are now in a position to formulate the main theorem of this section:

Theorem 3.1. Let $(Z, S)$ be an (infinite) strictly ergodic topological dynamical system, let $\mathcal{G}$ be a pathwise connected subgroup of the group of all homeomorphisms of $Y$, with the following property:

(A) For every nonempty open set $V$ in $Y$ and $\epsilon>0$ there are homeomorphisms $h_{1}, h_{2}, \ldots, h_{M}$ in $\mathcal{G}$ such that

$$
\frac{1}{M} \sum_{j=1}^{M} \mathbf{1}_{h_{j}(Y \backslash V)}(y) \leq \epsilon,
$$

for all $y \in Y\left(\mathbf{1}_{F}\right.$ denotes the characteristic function of a set $\left.F\right)$.

Then, for a generic (i.e., for a member of a residual subset) cocycle $G \in \overline{\operatorname{Cob}}(\mathcal{G})$, the corresponding extension $\left(X, T_{G}\right)$ of $(Z, S)$ is both minimal and isomorphic.

Remarks 3.2. $\quad$ 1. The assumptions imply that $Y$ contains nontrivial pathwise connected components, hence is uncountable.

2. The most obvious example of a space $Y$ and a group $\mathcal{G}$ satisfying these assumptions is the circle and the group of all orientation-preserving circle homeomorphisms. It is easy to see that the unit interval, even with its entire group of orientation preserving homeomorphisms, does not satisfy the condition $(A)$.

3. Clearly, the generic skew product, whose existence is claimed in this theorem, is an isomorphic but not an almost one-to-one extension of $(Z, S)$. There exists a function $\varphi: Z \rightarrow Y$ such that $z \mapsto(z, \varphi(z))$ is an isomorphism from $(Z, S)$ to $(X, T)$, and the unique invariant measure $\mu$ on $X$ is supported by the graph of $\varphi$. By minimality, this graph must be dense in $X=Z \times Y$.

4. Applied to minimal equicontinous systems $(Z, S)$, Theorem 3.1 answers negatively (although ineffectively) the Question 2.3 and hence also 1.6.

Proof. We need to demonstrate two claims: genericity of minimality and genericity of being an isomorphic extension. Of course, the intersection of two generic properties is still generic. Now, the first claim is proven in [12, Theorem 1] (notice that the condition $(A)$ implies that $\mathcal{G}$ acts minimally on $Y$ ). Alternatively, one can use [12, Theorem 2] (strict ergodicity includes minimality). Thus, it remains to show that isomorphic extensions form a residual subset of $\overline{\operatorname{Cob}}(\mathcal{G})$.

We begin with an outline of the proof. We will consider the relative product $X \underset{Z}{\times} X=\left\{\left(x, x^{\prime}\right) \in X \times X: \pi(x)=\pi\left(x^{\prime}\right)\right\}$, which in our case - where $X=Z \times Y$ is a product space - is homeomorphic to $\widetilde{X}:=Z \times Y \times Y$. Then, given $G \in C(Z, \mathcal{G})$, we define the corresponding cocycle extension as the map:

$$
\widetilde{T}_{G}\left(z, y_{1}, y_{2}\right)=\left(S z, G_{z}\left(y_{1}\right), G_{z}\left(y_{2}\right)\right) .
$$

We will show that for a generic $G \in \overline{\mathrm{Cob}}(\mathcal{G})$ this defines a uniquely ergodic map on $\widetilde{X}$. This will be done by a modification of the proof of [12, Theorem 2], and, in view of Proposition 2.5 this will complete our proof. 
We begin with a lemma, where the technical assumptions made on $\mathcal{G}$ are essential.

Lemma 3.3. Let $V$ be a nonempty open subset of $Y$, and fix $\gamma>0$. There exists a continuous map $t \mapsto h_{t}$ from $[0,1]$ into $\mathcal{G}$ such that for all $y \in Y$, with $\lambda$ denoting the Lebesgue measure on $[0,1]$, we have

$$
\lambda\left\{t \in[0,1]: h_{t}^{-1}(y) \notin V\right\}<\gamma .
$$

Proof. By assumption, there exist $\tilde{h}_{1}, \tilde{h}_{2}, \ldots, \tilde{h}_{M} \in \mathcal{G}$ satisfying the condition

$$
\frac{1}{M} \sum_{i=1}^{M} \mathbf{1}_{\tilde{h}_{i}(Y \backslash V)}(y) \leq \frac{\gamma}{2} .
$$

Define $h_{t}=\tilde{h}_{i}$ for $t \in\left[\frac{i-1}{M}+\frac{\gamma}{4 M}, \frac{i}{M}-\frac{\gamma}{4 M}\right](1 \leq i \leq M)$ and extend the map $t \mapsto h_{t}$, continuously to all of $[0,1]$ (here we use pathwise connectedness of $\mathcal{G}$ ). Now consider two cases for $y \in Y$ :

- If $y \notin \bigcup_{i=1}^{M} \tilde{h}_{i}(Y \backslash V)$, then, for every $t \in \bigcup_{i=1}^{M}\left[\frac{i-1}{M}+\frac{\gamma}{4 M}, \frac{i}{M}-\frac{\gamma}{4 M}\right]$, we have $h_{t}^{-1}(y) \in V$ and

$$
\lambda\left\{t \in I: h_{t}^{-1}(y) \notin V\right\} \leq 2 M \frac{\gamma}{4 M}=\frac{\gamma}{2} .
$$

- Otherwise there is a nonempty set $F \subset\{1, \ldots, M\}$ with $y \in \bigcap_{i \in F} \tilde{h}_{i}(Y \backslash V)$, and then

$$
\frac{|F|}{M}=\frac{1}{M} \sum_{i=1}^{M} \mathbf{1}_{\tilde{h}_{i}(Y \backslash V)}(y) \leq \frac{\gamma}{2}
$$

implying

$$
\lambda\left\{t \in I: h_{t}^{-1}(y) \notin V\right\} \leq \frac{|F|}{M}+2 M \frac{\gamma}{4 M} \leq \gamma
$$

We proceed with the main proof. For $f \in C(\widetilde{X},[0,1])$ and $\epsilon>0$ we denote

$$
E_{f, \epsilon}=\left\{G \in C(Z, \mathcal{G}): \exists c \in \mathbb{R}, \exists n \geq 1,\left\|\frac{1}{n+1} \sum_{k=0}^{n} f\left(\widetilde{T}_{G}^{k}\left(z, y_{1}, y_{2}\right)\right)-c\right\|<\epsilon\right\} .
$$

Clearly $E_{f, \epsilon}$ is open in $C(Z, \mathcal{G})$ and it is easy to check that $\mathcal{R}=\bigcap_{i, j \in \mathbb{N}} E_{f_{i}, 1 / j}$, where $\left\{f_{i}\right\}$ is a countable dense subset of $C(\widetilde{X},[0,1])$, consists precisely of the cocycles $G \in C(Z, \mathcal{G})$ for which $\left(\widetilde{X}, \widetilde{T}_{G}\right)$ is uniquely ergodic ${ }^{6}$. Thus, all we need to show is that for an arbitrary $f \in C(\widetilde{X},[0,1])$ and $\epsilon>0, E_{f, \epsilon} \cap \overline{\operatorname{Cob}}(\mathcal{G})$ is dense in $\overline{\operatorname{Cob}}(\mathcal{G})$, i.e., that $\operatorname{Cob}(\mathcal{G}) \subset \overline{E_{f, \epsilon}}$.

Let $H_{S}^{-1} H$ be a coboundary. We have

$$
H_{S}^{-1} H \in \overline{E_{f, \epsilon}} \Longleftrightarrow \mathrm{Id} \in H_{S} \overline{E_{f, \epsilon}} H^{-1}=\overline{H_{S} E_{f, \epsilon} H^{-1}},
$$

where $\mathrm{Id} \in C(Z, \mathcal{G})$ assigns to each $z \in Z$ the identity map $\operatorname{ld}_{Y}$ on $Y$ (the last equality follows from the fact that left and right multiplications by a fixed cocycle are homeomorphisms of $C(Z, \mathcal{G}))$. We need to identify the latter set.

\footnotetext{
${ }^{6}$ Formally, to guarantee unique ergodicity, in the condition defining $E_{f, \epsilon}$, in place of $\exists n \geq 1$ we should demand $\exists n_{0} \geq 1 \forall n \geq n_{0}$. However, it is not hard to see, that if one such $n$ exists, then an $n_{0}$, satisfying the correct condition, with a slightly larger $\epsilon$, can also be found (much larger than $n$ ).
} 
Define $\psi_{H}\left(z, y_{1}, y_{2}\right)=\left(z, H_{z}^{-1}\left(y_{1}\right), H_{z}^{-1}\left(y_{2}\right)\right)$. This is a self-homeomorphism of $\tilde{X}$. Thus, we have the obvious equality of uniform norms:

$$
\left\|\frac{1}{n+1} \sum_{k=0}^{n} f\left(\widetilde{T}_{G}^{k}\left(z, y_{1}, y_{2}\right)\right)-c\right\|=\left\|\frac{1}{n+1} \sum_{k=0}^{n} f\left(\widetilde{T}_{G}^{k}\left(\psi_{H}\left(z, y_{1}, y_{2}\right)\right)\right)-c\right\|,
$$

i.e., in the definition of $E_{f, \epsilon}$, we can replace the argument $\left(z, y_{1}, y_{2}\right)$ by $\psi_{H}\left(z, y_{1}, y_{2}\right)$.

A tedious but straightforward computation shows that

$$
\widetilde{T}_{H_{S}^{-1} G H}^{k}\left(\psi_{H}\left(z, y_{1}, y_{2}\right)\right)=\psi_{H}\left(\widetilde{T}_{G}^{k}\left(z, y_{1}, y_{2}\right)\right)
$$

Combined with the preceding observation, this easily implies that

$$
H_{S}^{-1} G H \in E_{f, \epsilon} \Leftrightarrow G \in E_{f \circ \psi_{H}, \epsilon}, \quad \text { i.e., } \quad H_{S} E_{f, \epsilon} H^{-1}=E_{f \circ \psi_{H}, \epsilon}
$$

(whence the closures are equal).

The equivalence (3.1) now means that for the required inclusion $\operatorname{Cob}(\mathcal{G}) \subset \overline{E_{f, \epsilon}}$ it suffices to show that $\mathrm{Id} \in \overline{E_{f \circ \psi_{H}, \epsilon}}$, for any $\epsilon>0$, any function $f \in C(\widetilde{X},[0,1])$, and any cocycle $H$. Since the family $\left\{f \circ \psi_{H}: f \in C(\tilde{X},[0,1]), H \in C(Z, \mathcal{G})\right\}$ equals $C(\tilde{X},[0,1])$, what we need is that for any $f \in C(\widetilde{X},[0,1])$ and $\epsilon>0$, we have Id $\in \overline{E_{f, \epsilon}}$. We will show a stronger fact: Id can be uniformly approximated by coboundaries from $E_{f, \epsilon}$. We formulate this as a lemma:

Lemma 3.4. Given $f \in C(\widetilde{X},[0,1]), \epsilon>0$ and $\delta>0$, there exists a coboundary $G=H_{S}^{-1} H \in E_{f, \epsilon}$ satisfying $d(G, \mathrm{Id})<\delta$, (here $d$ denotes the uniform distance on $C(Z, \mathcal{G})$ ).

Proof. Observe that the expression in the (modified) definition of $E_{f, \epsilon}$,

$$
\frac{1}{n+1} \sum_{k=0}^{n} f\left(\widetilde{T}_{G}^{k}\left(\psi_{H}\left(z, y_{1}, y_{2}\right)\right)=\frac{1}{n+1} \sum_{k=0}^{n} f\left(S^{k} z, H_{S^{k} z}^{-1}\left(y_{1}\right), H_{S^{k} z}^{-1}\left(y_{2}\right)\right)\right.
$$

equals the ergodic average, under the action of $S$, of the real-valued function (of one variable, with two parameters) $f_{y_{1}, y_{2}} \in C(Z,[0,1])$ given by

$$
f_{y_{1}, y_{2}}(z)=f\left(z, H_{z}^{-1}\left(y_{1}\right), H_{z}^{-1}\left(y_{2}\right)\right) .
$$

Strict ergodicity of $(Z, S)$ implies that if we fix $\left(y_{1}, y_{2}\right) \in Y^{2}$, then these averages tend uniformly over $z \in Z$ to the integral

$$
\int f_{y_{1}, y_{2}} d \nu(z)
$$

(recall that $\nu$ is the unique invariant measure on $Z$ ). Since the map $\left(y_{1}, y_{2}\right) \mapsto f_{y_{1}, y_{2}}$ is obviously continuous, the family of functions $\left\{f_{y_{1}, y_{2}}:\left(y_{1}, y_{2}\right) \in Y^{2}\right\}$ is compact and thus the above convergence is uniform also over $\left(y_{1}, y_{2}\right) \in Y^{2}$.

So, the condition $G \in E_{f, \epsilon}$ in the lemma is equivalent to

$$
\left|\int f\left(z, H_{z}^{-1}\left(y_{1}\right), H_{z}^{-1}\left(y_{2}\right)\right) d \nu(z)-c\right|<\epsilon
$$

for some constant $c$ and all $\left(y_{1}, y_{2}\right) \in Y^{2}$.

We let $V \subset Y$ be an open set such that

$$
\sup \left|f\left(z, v_{1}, v_{2}\right)-f\left(z, v_{1}^{\prime}, v_{2}^{\prime}\right)\right|<\frac{\epsilon}{2},
$$


where the supremum ranges over all $z \in Z$ and $v_{1}, v_{2}, v_{1}^{\prime}, v_{2}^{\prime} \in V$, and set $\gamma=\frac{\epsilon}{16}$. Lemma 3.3 provides a continuous assignment $t \mapsto h_{t}$ with the appropriate properties.

We can now proceed with the construction of $H$ (and thus $G$ ). Let $\eta>0$ be such that

$$
\left|t_{1}-t_{2}\right|<\eta \Longrightarrow d\left(h_{t_{1}}^{-1} h_{t_{2}}, \mathrm{Id}_{Y}\right)<\delta
$$

and fix an $N \in \mathbb{N}$ with $\frac{1}{N}<\min \left\{\frac{\eta}{2}, \gamma\right\}$. There exists a measurable subset $K$ of $Z$ with $\nu(K)>0$, such that the images $K, S(K), \ldots, S^{N^{2}-1}(K)$ are pairwise disjoint while the measure of their union exceeds $1-\gamma$. By regularity, we can assume that $K$ is a closed set, moreover, we can arrange that it is homeomorphic to the Cantor set. Let $\tilde{\theta}: K \rightarrow[0,1]$ be a continuous surjection for which the push-forward measure $\tilde{\theta}_{*}(\nu \mid K)$ equals $\lambda$, where $\nu \mid K$ denotes the (normalized) conditional measure on $K$ obtained from $\nu$ (such a surjection exists for any continuous probability measure on the Cantor set). Extend $\tilde{\theta}$ to $\bigcup_{i=0}^{N^{2}-1} S^{i}(K)$ by:

$$
\tilde{\theta}(z)=\tilde{\theta}\left(S^{-i} z\right) \text { if } z \in S^{i}(K) \quad\left(i=1, \ldots, N^{2}-1\right)
$$

and then extend it again to a continuous map $\tilde{\theta}: Z \rightarrow[0,1]$. Finally, for every $z \in Z$, put

$$
\theta(z)=\frac{1}{N} \sum_{i=0}^{N-1} \tilde{\theta}\left(S^{-i} z\right)
$$

and define $H \in C(Z, \mathcal{G})$ by setting $H_{z}=h_{\theta(z)}$. It remains to verify the required properties of $G=H_{S}^{-1} H$.

We claim that $G \in E_{f, \epsilon}$, namely, that for every $y_{1}, y_{2} \in Y$, we have

$$
\left|\int f\left(z, H_{z}^{-1}\left(y_{1}\right), H_{z}^{-1}\left(y_{2}\right)\right) d \nu(z)-c\right|<\epsilon
$$

(i.e., (3.2) holds), where $c=\int f(z, v, v) d \nu(z)$, with $v$ being any point selected from the open set $V \subset Y$. Indeed,

$$
\begin{aligned}
& \int\left|f\left(z, H_{z}^{-1}\left(y_{1}\right), H_{z}^{-1}\left(y_{2}\right)\right)-f(z, v, v)\right| d \nu(z) \leq \\
& \int_{\bigcup_{i=0}^{N^{2}-1} S^{i}(K)}\left|f\left(z, H_{z}^{-1}\left(y_{1}\right), H_{z}^{-1}\left(y_{2}\right)\right)-f(z, v, v)\right| d \nu(z)+2\|f\| \gamma \leq \\
& \int_{\bigcup_{i=0}^{N^{2}-N-1} S^{i}(K)}\left|f\left(z, H_{z}^{-1}\left(y_{1}\right), H_{z}^{-1}\left(y_{2}\right)\right)-f(z, v, v)\right| d \nu(z)+2\|f\|(\gamma+N \cdot \nu(K))< \\
& \sum_{i=0}^{N^{2}-N-1} \int_{K}\left|f\left(S^{i} z, H_{S^{i} z}^{-1}\left(y_{1}\right), H_{S^{i} z}^{-1}\left(y_{2}\right)\right)-f(z, v, v)\right| d \nu(z)+4 \gamma= \\
& \sum_{i=0}^{N^{2}-N-1} \int_{K}\left|f\left(S^{i} z, H_{z}^{-1}\left(y_{1}\right), H_{z}^{-1}\left(y_{2}\right)\right)-f(z, v, v)\right| d \nu(z)+4 \gamma,
\end{aligned}
$$

(we have used the fact that for $z \in K, H_{z}=H_{S z}=\cdots=H_{S^{N^{2}-N-1} z}$, and the inequalities $\nu(K) \leq \frac{1}{N^{2}}, \frac{1}{N}<\gamma$ and $\|f\| \leq 1$ ). Next, consider a point $z \in K$ for which both $h_{\theta(z)}^{-1}\left(y_{1}\right) \in V$ and $h_{\theta(z)}^{-1}\left(y_{2}\right) \in V$. By the choice of $V$, for such $z$, the integrand does not exceed $\frac{\epsilon}{2}$. On the other hand, a point $z \in K$ does not fulfill this condition 
only when $t=\theta(z)$ is such that either $y_{1}$ or $y_{2}$ fall outside $h_{t}(V)$. The measure $\lambda$ of such $t$ 's is at most $2 \gamma$ (this is what Lemma 3.3 yields), i.e., the conditional measure $\nu \mid K$ of such $z$ 's is at most $2 \gamma$. We conclude that the last line above is bounded by

$$
\left(N^{2}-N\right) \nu(K)\left(\frac{\epsilon}{2}+4 \gamma\right)+4 \gamma \leq \frac{\epsilon}{2}+8 \gamma \leq \epsilon,
$$

as claimed (we have used one more time the fact that $\nu(K) \leq \frac{1}{N^{2}}$ ).

Finally, in order to show that $d\left(H_{S z}^{-1} H_{z}, \mathrm{Id}_{Y}\right)<\delta$, for every $z \in Z$, note that, because $\theta(z)$ is defined as an $N$-step ergodic average, we have $|\theta(S z)-\theta(z)| \leq \frac{2}{N}<$ $\eta$, which, by the choice of $\eta$, yields $d\left(H_{S z}^{-1} H_{z}, \mathrm{Id}_{Y}\right)=d\left(h_{\theta(S z)}^{-1} h_{\theta(z)}, \mathrm{Id}_{Y}\right)<\delta$. This concludes the proof of Lemma 3.4.

The proof of Theorem 3.1 is now complete.

Remark 3.5. The same proof will show that for a residual set of $G$ 's in $\overline{\operatorname{Cob}}(\mathcal{G})$, the analogous relative product action on $Z \times Y \times Y \times \cdots \times Y$ ( $k$ times) is uniquely ergodic, and hence also that the infinite product will be uniquely ergodic for a residual set of G's.

\section{Some CONCRETE EXAMPles AND RELATED RESUltS}

As in [12] we can single out the following concrete examples. Let $(Y, \mathcal{G})$ denote $\left(\mathbb{P}^{n}, S L(n+1, \mathbb{R})\right)$ or $(Q, \mathcal{G})$ where $\mathcal{G}$ is the identity path component of $\mathcal{H}(Q)$, the group of homeomorphisms of $Q$. Here $\mathbb{P}^{n}$ and $Q$ denote the $n$-dimensional projective space and the Hilbert cube respectively. It is easy to check that, in both cases, the conditions in Theorem 3.1 hold (see [12]). Clearly the action of $S L(n+1, \mathbb{R})$ on $\mathbb{P}^{n}$ is (literally) transitive and this is also true for the action of $\mathcal{G}$ on $Q$; in particular these actions are minimal. Thus for an arbitrary infinite, minimal, uniquely ergodic, metric system $(Z, S)$, for a residual set of $G$ 's in $\overline{\operatorname{Cob}}(\mathcal{G})$, the corresponding homeomorphisms of $X=Z \times Y$, where $Y=\mathbb{P}^{n}$ or $Q$, is strictly ergodic. Moreover, denoting by $\mu$ the unique invariant measure on $X$ and by $\nu$ the unique invariant measure on $Z$, by Theorem 3.1, the extension map $\pi:\left(X, \mu, T_{G}\right) \rightarrow(Z, \nu, S)$ is a measuretheoretic isomorphism; i.e. $\pi$ is an isomorphic extension. In particular if we let $(Z, S)=\left(\mathbb{T}, R_{\alpha}\right)$, an irrational rotation of the circle, and $(Y, \mathcal{G})=\left(\mathbb{P}^{1}, S L(2, \mathbb{R})\right)$, then, since $\mathbb{P}^{1}$ is homeomorphic to $\mathbb{T}$, we can obtain these minimal systems on the torus $\mathbb{T}^{2}$.

Recall that a metric dynamical system is called tame when its enveloping semigroup has cardinality $\leq 2^{\aleph_{0}}$. Both $(Z, S)=\left(\mathbb{T}, R_{\alpha}\right)$ and $(Y, \mathcal{G})=\left(\mathbb{P}^{n}, S L(n+1, \mathbb{R})\right)$ are tame (for more details see [4], [1] and [11, Example 8.31(6)]). However, none of the minimal systems $\left(X, T_{G}\right)=\left(Z \times Y, T_{G}\right)$ given by Theorem 3.1 is tame. In fact, a theorem of Huang [15], Kerr \& Li [17], and Glasner [10] asserts that a tame minimal dynamical system is necessarily almost automorphic, i.e., an almost one-to-one extension of its maximal equicontinuous factor, and that this extension is isomorphic. More precisely, as quoted from [10, Theorem 5.1], we have:

Theorem 4.1. Let $\Gamma$ be an Abelian group and $(X, \Gamma)$ a metric tame minimal system. Then: 
1. The system $(X, \Gamma)$ is almost automorphic. Thus there exist:

(a) A compact topological group $Y$ with Haar measure $\eta$, and a group homomorphism $\kappa: \Gamma \rightarrow Y$ with dense image.

(b) A homomorphism $\pi:(X, \Gamma) \rightarrow(Y, \Gamma)$, where the $\Gamma$ action on $Y$ is via $\kappa$.

(c) The sets $X_{0}=\left\{x \in X: \pi^{-1}(\pi(x))=\{x\}\right\}$ and $Y_{0}=\pi\left(X_{0}\right)$ are dense $G_{\delta}$ subsets of $X$ and $Y$ respectively.

2. The system $(X, \Gamma)$ is uniquely ergodic with unique invariant measure $\mu$ such that with $\pi_{*}(\mu)=\eta$, the map $\pi:(X, \mu, \Gamma) \rightarrow(Y, \eta, \Gamma)$ is a measure-theoretic isomorphism of the corresponding measure preserving systems.

By a theorem of Furstenberg and Kesten [6], for an ergodic system $(Z, \nu, S)$ and a measurable map (cocycle) $\rho: Z \rightarrow S L(n, \mathbb{R})$, such that both $\log ^{+}\|\rho(z)\|$ and $\log ^{+}\left\|\rho^{-1}(z)\right\|$ are integrable functions, the Lyaponov limit

$$
\Lambda(\rho)=\lim \frac{1}{n+1} \log \left\|\rho\left(T^{n} z\right) \cdots \rho(T z) \rho(z)\right\|
$$

exists $\nu$ almost everywhere. In the case where $(Z, S, \nu)$ is a strictly ergodic system and $\Lambda(\rho)=0$, it was shown by Furman [7] that the limit exists uniformly on $Z$. However, in general, even in the case where $(Z, S, \nu)$ is strictly ergodic, as was shown by Walters [23] and Herman [14], the convergence need not be uniform.

In the two-dimensional case; i.e. when $\rho$ maps into $S L(2, \mathbb{R})$, Furman considers the associated skew product $X=Z \times Y$, where $Y=\mathbb{P}^{1}$ and then, using Oseledec' theorem [20], he proves the following:

Theorem 4.2. Let $(Z, S, \nu)$ be a strictly ergodic system, $\rho: Z \rightarrow S L(2, \mathbb{R})$ a continuous cocycle, and let $(X, T)=\left(Z \times \underset{\rho}{\mathbb{P}^{1}}, T\right)$ be the corresponding skew-product. Then there are three possibilities:

(1) $\Lambda(T):=\Lambda(\rho)=0$, in which case the convergence in (4.1) is uniform and $(X, T)$ is uniquely ergodic.

(2) $\Lambda(\rho)>0$. There are on $X$ exactly two ergodic measures $\theta^{+}$and $\theta^{-}$. They are graph measures, i.e. carried by the graphs of two measurable functions $u^{ \pm}: Z \rightarrow \mathbb{P}^{1}$

(2a) On $(X, T)$ there is a unique minimal set $M$, with $M=\operatorname{supp} \theta^{+}=\operatorname{supp} \theta^{-}$, and there is a dense $G_{\delta}$ subset $Z_{0} \subset Z$ on which

$$
\liminf \frac{1}{n+1} \log \left\|\rho\left(T^{n} z\right) \cdots \rho(T z) \rho(z)\right\|<\Lambda(\rho) .
$$

(2b) On $X$ there are exactly two minimal sets $M^{+}$and $M^{-}$, which are the graphs of continuous functions $u^{ \pm}: Z \rightarrow \mathbb{P}^{1}$. Each of these minimal sets supports a unique ergodic measure, $\theta^{+}$and $\theta^{-}$with $M^{+}=\operatorname{supp} \theta^{+}$and $M^{-}=\operatorname{supp} \theta^{-}$, and again the convergence in (4.1) is uniform.

Examples of the type (2a) were given by Walters [23] and Herman [14]. Furman also shows that a cocycle $\rho$ of type $(2 \mathrm{~b})$ is continuously diagonalizable and continuously cohomologous to a function whose values eventually lie in the set of positive matrices. For more details see [7]. It follows from this theorem that the strictly ergodic systems on $Z \times \mathbb{P}^{1}$ whose existence is shown in [12, Theorem 2] and in Theorem 3.1 above are all of the type $\Lambda(T)=0$. 


\section{REFERENCES}

[1] Ethan Akin, Enveloping linear maps, in: Topological dynamics and applications, Contemporary Mathematics 215, a volume in honor of R. Ellis, 1998, pp. 121-131.

[2] Joseph Auslander, Minimal flows and their extension, North-Holland, 1988.

[3] T. Downarowicz, S. Kasjan, Odometers and Toeplitz subshifts revisited in the context of Sarnaks conjecture, preprint, arXiv:1502.02307.

[4] Robert Ellis, The enveloping semigroup of projective flows, Ergod. Th. Dynam. Sys. 13 (1993), 635-660.

[5] Harry Furstenberg, Recurrence in ergodic theory and combinatorial number theory, Princeton university press, Princeton, N.J., 1981.

[6] Harry Furstenberg and Harry Kesten, Products of random matrices, Ann. Math. Stat. 31, (1960), 457-489.

[7] Alex Furman, On the multiplicative ergodic theorem for uniquely ergodic systems, Ann. Inst. H. Poincar Probab. Statist. 33, (1997), 797-815.

[8] Felipe Garcia-Ramos, Weak forms of topological and measure theoretical equicontinuity: relationships with discrete spectrum and sequence entropy, Ergod. Th. and Dynam. Sys. to appear.

[9] Eli Glasner, Ergodic theory via joinings, AMS, Surveys and Monographs, 101, 2003.

[10] Eli Glasner, The structure of tame minimal dynamical systems, Ergod. Th. and Dynam. Sys. 27, (2007), 1819-1837.

[11] Eli Glasner and Michael Megrelishvili, Representations of dynamical systems on Banach spaces, in Recent progress in general topology. III, 399-470, Atlantis Press, Paris, 2014.

[12] Eli Glasner and Benjamin Weiss, On the construction of minimal skew-products, Israel J. of Math. 34, (1979), 321-336.

[13] Eli Glasner and Benjamin Weiss, On the interplay between measurable and topological dynamics. Handbook of dynamical systems. Vol. 1B, 597-648, Elsevier B. V., Amsterdam, 2006.

[14] M. R. Herman, Construction d'un diffomorphisme minimal d'entropie topologique non nulle. (French) [Construction of a minimal diffeomorphism with nonzero topological entropy], Ergod. Th. \& Dynam. Sys. 1 (1981), 65-76.

[15] Wen Huang, Tame systems and scrambled pairs under an abelian group action, Ergod. Th. Dynam. Sys. 26 (2006), 1549-1567.

[16] W. Huang, P. Lu, and X. Ye. Measure-theoretical sensitivity and equicontinuity, Israel Journal of Mathematics 183 (2011), 233-283.

[17] David Kerr and Hanfeng Li, Independence in topological and $C^{*}$-dynamics, Math. Ann. 338, (2007), 869-926.

[18] E. Lehrer Topological Mixing and Uniquely Ergodic Systems, Israel J. of Math. 57 (1987), 239-255

[19] J. Li, S. Tu, X. Ye, Mean equicontinuity and mean sensitivity, arXiv:1312.7663, to appear in Ergodic Theory and Dynamical Systems.

[20] V. I. Oseledec, A multiplicative ergodic theorem. Lyaponov characteristic numbers for dynamical systems Trans. Moscow Mat. Soc., 19, (1968), 197-231.

[21] A. Rosenthal, Strictly ergodic models for noninvertible transformations. Israel J. Math. 64, (1988), 57-72.

[22] P. Sarnak, Three Lectures on the Möbius Function Randomness and Dynamics http://www.math.ias.edu/files/wam/2011/PSMobius.pdf

[23] Peter Walters, Unique ergodicity and random matrix products. Lyapunov exponents (Bremen, 1984), 37-55, Lecture Notes in Math., 1186, Springer, Berlin, 1986.

[24] Benjamin Weiss, Strictly ergodic models for dynamical systems, Bull. of the Amer. Math. Soc. 13, (1985), 143-146.

[25] W. A. Veech, The equicontinuous structure relation for minimal abelian transformation groups, Amer. J. Math. 90, (1968), 723-732. 
Institute of Mathematics, Polish Academy of Science, Śniadeckich 8, 00-656 WarSAW, POLAND

E-mail address: downar@pwr.edu.pl

Department of Mathematics, Tel Aviv University, Tel Aviv, Israel

E-mail address: glasner@math.tau.ac.il 